\title{
Stability of anthocyanins in vitro
}

\author{
A. M. Amini, A. Rodriguez-Mateos, J. P. E. Spencer and P. Yaqoob \\ Department of Food and Nutritional Sciences, The University of Reading, Whiteknights PO Box 226, Reading RG6 6AP
}

Anthocyanins (ACNs) are abundant in berry fruits and are associated with a variety of health benefits to humans, including cardiovascular disease prevention, improved glucose metabolism, body weight regulation and improvement of vision and memory ${ }^{(1)}$. However, ACNs are known to be unstable in vitro ${ }^{(2)}$ and this needs to be considered in the design of cell studies. The aim of this experiment was to investigate the stability of cyanidin-3-glucoside $(\mathrm{C} 3 \mathrm{G})$ and cyanidin-3-rutinoside $(\mathrm{C} 3 \mathrm{R})$ in vitro over a $24 \mathrm{~h}$ period.

The methodological design was adapted from a study by Woodward et al. ${ }^{(2)}$. ACNs (C3G and C3R) at $10 \mu \mathrm{M}$ in either RPMI medium alone or in RPMI medium with added foetal calf serum (FCS) and Penicillin-Streptomycin (AB) were incubated at $37^{\circ} \mathrm{C}$ in a $5 \% \mathrm{CO}_{2}$ atmosphere. After 0, 0.5, 2, 4, 6, 12 and $24 \mathrm{~h}$, aliquots were removed, acidified with formic acid, filtered and analysed using high-pressure liquid chromatography (HPLC). To evaluate the influence of freezing, half of the acidified aliquots were frozen at $-80^{\circ} \mathrm{C}$ for 2 to 3 weeks prior to HPLC analysis.
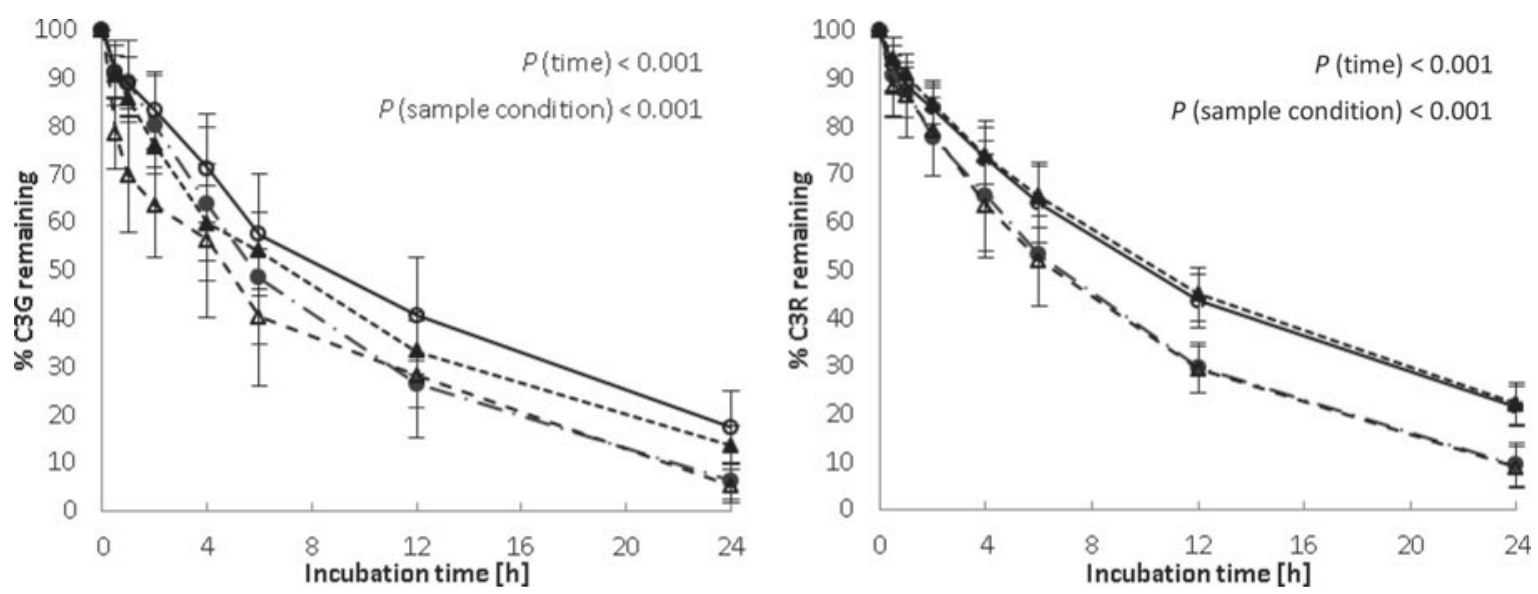

Effects of culture and storage conditions on stability of cyanidin-3-glucoside (C3G) and cyanidin-3-rutinoside (C3R). RPMI fresh (-๑-), RPMI frozen (- - $\triangle$ - -), RPMI + FCS + AB Fresh (-O-), RPMI + FCS + AB Frozen (- $-\boldsymbol{\Delta}--)$. Data are mean \pm sD for $n=5$ independent experiments, with all analyses being conducted in quintuplicate. SPSS 21 was used to analyse effects of time and sample condition (two-way ANOVA) and effects of sample condition per timepoint (one-way ANOVA with Tukey post hoc tests).

There was significant degradation of $\mathrm{C} 3 \mathrm{G}$ and $\mathrm{C} 3 \mathrm{R}$ over time and a significant effect of sample condition $(P<0.001$; two-way ANOVA). For both compounds, approximately $50 \%$ of the initial dose had disappeared by $6 \mathrm{~h}$ and only 5 to $20 \%$ of baseline levels were still detectable after incubation for $24 \mathrm{~h}$ (see Figure). For C3G, sample condition significantly affected stability at $1 \mathrm{~h}$ and $24 \mathrm{~h}(P<0.01$; oneway ANOVA), such that incubation in RPMI alone for $1 \mathrm{~h}$ followed by freezing reduced stability compared with all other conditions $(P<0.05$; post-hoc Tukey tests). At $24 \mathrm{~h}$, inclusion of FCS and AB in the medium improved stability of C3G compared with RPMI alone ( $P<0.02$ for fresh and $P<0.01$ for frozen samples), but freezing samples per se before analysis did not affect stability. For C3R, sample condition only affected stability at $24 \mathrm{~h}(P<0.001$ one-way ANOVA), such that FCS and AB improved stability in both freshly analysed and frozen samples $(P<0.01$; post-hoc Tukey tests). In conclusion, given the poor stability of ACNs it is prudent to conduct assays with short incubation periods when their biological properties are determined in cell culture studies. Freezing samples of medium for later ACN analysis only accelerated degradation at the $1 \mathrm{~h}$ timepoint and FCS/AB does not prevent degradation before $24 \mathrm{~h}$.

This work was supported by a BBSRC CASE studentship with GlaxoSmithKline as industrial sponsor.

1. Tsuda T (2012) Mol Nutr Food Res 56, 159-170.

2. Woodward G, Kroon P, Cassidy A et al. (2009) J Agric Food Chem 57, 5271-5278. 